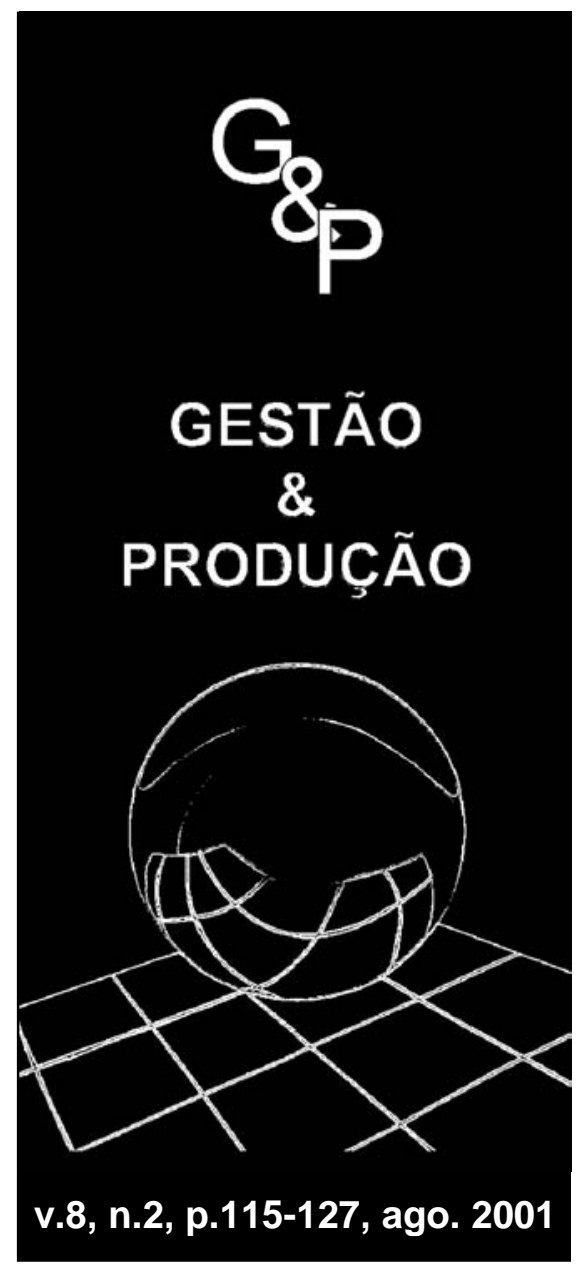

\title{
UM MODELO ESTOCÁSTICO PARA A MANUTENÇÃO DE UM EQUIPAMENTO BASEADO NA INSPEÇÃO DAS PEÇAS PRODUZIDAS
}

\author{
Mônica Maria De Marchi \\ Solon Venâncio de Carvalho \\ Laboratório Associado de Computação \\ e Matemática Aplicada (LAC) \\ Instituto Nacional de Pesquisas Espaciais (INPE) \\ Caixa Postal 515, 12201-970 - São José dos Campos - SP \\ E-mail: solon@lac.inpe.br \\ Paulo Renato de Morais \\ Instituto de Pesquisa e Desenvolvimento (IP\&D) \\ Universidade do Vale do Paraíba (UNIVAP) \\ Av. Shishima Hifumi, 2911 - Urbanova, 12244-000 \\ São José dos Campos - SP \\ E-mail: prenato@univap.br
}

\section{Resumo}

Apresenta-se um modelo de manutenção para um equipamento que se deteriora com o tempo. $O$ equipamento produz periodicamente um lote de peças. Cada lote produzido é inspecionado e, em função do número de peças defeituosas encontradas, deve-se decidir sobre deixar o equipamento operando ou revisá-lo. O objetivo é obter uma política de manutenção que minimize o custo médio a longo prazo de operação e revisão do equipamento. A estrutura de custos inclui um custo de operação, um custo de revisão e um custo por peça defeituosa produzida. Para obter uma política de manutenção de custo mínimo, o problema é modelado como um Processo Markoviano de Decisão com Informação Parcial. Exemplos numéricos são apresentados.

Palavras-chave: sistema de manutenção, política de revisão, processo markoviano de decisão com informação parcial.

\section{Introdução}

obtenção de modelos de manutenção para
problema clássico na literatura. Emáa é ulgumas
situações reais, o nível de deterioração do sistema não pode ser observado diretamente. Nestes casos, podem ser utilizados diferentes mecanismos para obtenção de informações sobre este nível. Encontram-se, por exemplo, trabalhos como o de TAPIERO (1987), nos quais a qualidade de um produto é uma função explícita 
do nível de produção e das atividades relacionadas à qualidade até aquele momento. Nos trabalhos de MILIONI \& PLISKA (1988) e OZEKICI \& PLISKA (1991), para se encontrar o nível de deterioração do sistema, um teste cujo resultado é do tipo binário é realizado. Em GONG \& TANG (1997), a informação sobre o nível de deterioração é obtida por sensores.

Neste trabalho, a informação sobre o nível de deterioração do equipamento é obtida pela inspeção de sua produção. A idéia é mostrar que, a partir do resultado dessa inspeção, pode-se tomar decisões sobre quando revisá-lo. Isto é importante, por exemplo, em situações em que o custo de inspeção do equipamento é elevado ou esta inspeção não pode ser executada por algum motivo.

$\mathrm{Na}$ literatura, problemas do tipo proposto neste trabalho, estão relacionados a modelos de decisão com informação parcial sobre os estados do processo e são conhecidos como Processos Markovianos de Decisão com Informação Parcial. Estes modelos podem ser vistos como casos particulares de Processos Markovianos de Decisão Parcialmente Observáveis, que começaram a ser tratados computacionalmente a partir de trabalhos desenvolvidos, por exemplo, por SMALLWOOD \& SONDIK (1973). O algoritmo, por eles proposto, encontra políticas ótimas para problemas de horizonte finito e soluções perto do ótimo para problemas de horizonte infinito. Segundo CASSANDRA (1994), este algoritmo, além de difícil compreensão e implementação, quando utilizado na resolução de problemas de grande porte incorre em um tempo de processamento muito grande. Outros trabalhos surgiram com diferentes abordagens, como destacado por MONAHAN (1982) e LOVEJOY (1991) com o objetivo de solucionar este problema.

O que diferencia um modelo considerando um sistema como parcialmente observável de um modelo considerando um sistema com informação parcial é como são obtidas as políticas de controle que determinarão as ações a serem tomadas. Em um Processo Markoviano de
Decisão Parcialmente Observável, as ações prescritas por uma política de controle ótima em geral dependem de informações contidas no histórico do processo. Já em um Processo Markoviano de Decisão com Informação Parcial, busca-se uma "boa" política dentro da classe das políticas markovianas, onde para cada observação associa-se uma ação.

Para obter uma política de manutenção de custo mínimo, o sistema, neste trabalho, será modelado por um Processo Markoviano de Decisão e resolvido utilizando-se o algoritmo HL, apresentado em HORDIJK \& LOEVE (1994). Este algoritmo obtém políticas markovianas subótimas para modelos a tempo discreto sob o critério do custo esperado médio a longo prazo. Por ser do tipo de aproximações sucessivas, este algoritmo é de fácil implementação computacional.

O modelo estudado é uma extensão de alguns trabalhos existentes na literatura que tratam de problemas de manutenção de equipamentos. Em LUSS (1976), por exemplo, políticas de reparo preventivos ou de inspeção são obtidas observando o grau de deterioração do equipamento. Em ROSENFIELD (1976 a, b), a idéia da informação imperfeita sobre o estado do processo é introduzida e, assim como em LUSS (1976), considera-se um custo de inspeção para determinar o exato estado do processo. Neste modelo considera-se que a informação sobre o nível de deterioração do equipamento é obtida inspecionando os lotes de peças produzidos. Com relação ao trabalho desenvolvido por ROSS (1971), o estado do sistema é conhecido através da amostragem nos itens produzidos, enquanto que neste trabalho toda a produção é avaliada.

A organização deste trabalho segue a seguinte estrutura: na Seção 2, apresenta-se a formulação e hipóteses do modelo; na Seção 3, apresenta-se a implementação e o algoritmo para o modelo com informação parcial; na Seção 4, resultados numéricos são apresentados; e, na Seção 5, são feitos alguns comentários finais sobre o trabalho. 


\section{Descrição do Modelo}

Considera-se um equipamento que sofre um processo de deterioração com o tempo segundo um processo markoviano. Este equipamento produz periodicamente lotes de peças de tamanho $N$. Por hipótese, não se admite quebra do equipamento durante a produção. Assim que um lote de peças é produzido, ele é enviado para inspeção. $O$ tempo de inspeção de um lote é considerado desprezível. Cada peça produzida é classificada como boa ou defeituosa.

Admite-se que o nível de deterioração do equipamento não pode ser observado. Por isso, o número de peças defeituosas encontradas num lote será usado para decidir sobre manter o equipamento operando ou revisá-lo. O tempo de revisão do equipamento é considerado igual ao tempo de produção de um lote de peças. Após cada revisão, o equipamento é considerado novo.

Os instantes de observação do equipamento ocorrem após a inspeção de um lote ou no final de uma revisão. Nestes instantes, uma decisão deve ser tomada. Quando se decide manter o equipamento operando, incorre-se em um custo de operação, dependente do nível de deterioração do equipamento, e em um custo fixo de produção de peça defeituosa. Quando se decide revisar o equipamento, incorre-se em um custo de revisão dependente do nível de deterioração do equipamento.

O processo de deterioração do equipamento é um processo markoviano a tempo discreto caracterizado pela hipótese de que a probabilidade de produção de uma peça defeituosa e as próximas deteriorações do equipamento dependem apenas do nível corrente de deterioração e pela hipótese de que o tempo de produção de um lote de tamanho $N$ e o tempo de revisão são considerados fixos e iguais.

Assim, em um determinado instante de decisão, o sistema pode estar em um dos seguintes níveis de deterioração, $E_{I}=\{0,1,2, \ldots, D\}$, onde o estado $O$ significa sistema novo, os estados $1,2, \ldots, D-1$ representam graus crescentes de deterioração causados pelo uso e o estado $D$ significa o estado final. $\mathrm{O}$ estado $D$ não é somente o nível máximo de deterioração, pode significar, também, um mau funcionamento que leva a condições de operações insatisfatórias.

Apesar de se supor conhecido (ou estatisticamente estimado) o processo de deterioração do sistema, por hipótese, não pode ser observado diretamente em cada instante de decisão. Então, o sistema é observado parcialmente através de um mecanismo de observação, $E_{\Phi}$, que, no final de cada período, fornece como informação as seguintes observações: $E_{\Phi}=\{0,1,2, \hbar, N, \psi\}$, onde os valores de $O$ a $N$ são informações fornecidas pela inspeção da produção, 0 significando que toda a produção está perfeita e $N$ que toda a produção está com defeito. $\psi$ é um valor arbitrário que informa o final de uma revisão do sistema.

O espaço de ações é definido por $A=\{0,1\}$, onde 0 significa manter o equipamento em operação e 1 significa revisá-lo.

A evolução do estado do equipamento ao longo do tempo, como um Processo Markoviano de Decisão com Informação Parcial, depende do número de peças defeituosas encontradas pela inspeção, $n \in E_{\Phi}$, e da decisão tomada, $a \in A$. Neste sentido, é necessário o cálculo dos seguintes parâmetros:

$p_{i j}(a)=$ probabilidade de no próximo instante de observação o sistema interno estar no estado $j \in E_{I}$, dado que o sistema interno está no estado $i \in E_{I}$ e a ação $a \in A$ é escolhida;

$q_{i n}(a)=$ probabilidade de se observar $n \in E_{\Phi}$, dado que o estado interno do sistema é $i \in E_{I}$ e a ação $a \in A$ é tomada;

$c_{i}(a)=$ valor esperado do custo total incorrido até o próximo instante de observação dado que o sistema está no estado $i \in E_{I}$ e a ação $a \in A$ é escolhida.

As observações sobre o estado do equipamento ocorrem no final de cada revisão ou após a inspeção de um lote de peças. A decisão sobre a revisão do equipamento é baseada apenas no número de peças defeituosas encontradas no último lote produzido, o que caracteriza uma 
tomada de decisão com informação parcial sobre o estado do equipamento, pois o nível de deterioração é considerado não-observável. Este modelo é denominado ao longo do texto como modelo com informação parcial.

Para a obtenção da probabilidade de transição dos estados internos do sistema, $p_{i j}(a)$, considera-se que se a ação escolhida for manter o sistema em funcionamento $(a=0)$, em um determinado estado interno $i$, a probabilidade de produzir um produto defeituoso, $\varphi(i)$, é dependente apenas do nível de deterioração $i$. Assim, estando em um estado interno $i$, após ter produzido, o novo estado será $j$, com probabilidade $p_{d}(i, j)$, onde $p_{d}(i, j)=0$ se $j<i$ e $\sum_{j=i}^{D} p_{d}(i, j)=1$ se $j \geq i$, para todo $i, j \in E_{I}$. Por outro lado, quando decide-se por revisar o equipamento $(a=1)$, o novo estado interno será $j=0 \in E_{I}$, com probabilidade 1 . Neste caso, o equipamento é considerado novo e nenhum lote foi produzido. Todas as probabilidades são supostas conhecidas. Assim, tem-se que:

$$
p_{i j}(a)= \begin{cases}p_{d}(i, j) & \text { se } a=0 \\ 1 & \text { se } a=1 \\ 0 & \text { caso contrário }\end{cases}
$$

para todo $i, j \in E_{I}$.

Para obtenção da matriz de observação, quando a observação ocorre após uma inspeção, as decisões possíveis são manter o equipamento operando ou revisá-lo. Esta decisão é baseada na informação fornecida pela inspeção, que é representada por uma binomial com parâmetros $N$ e $\varphi(i)$, onde $n$ (para $n=0,1,2, \ldots, N \in E_{\Phi}$ ), representa o número de produtos com defeitos fornecidos pela inspeção e $\varphi(i)$ é a probabilidade de se produzir um produto com defeito no nível de deterioração $i$.

Quando a observação ocorre após a realização de uma revisão, isto é, quando o estado de observação for $n=\psi \in E_{\Phi}$, para qualquer estado interno do sistema $i \in E_{I}$ a única ação possível é continuar a produzir $(a=0)$. Assim, tem-se que:

$$
q_{\text {in }}(a)=\left\{\begin{array}{l}
\left(\begin{array}{l}
N \\
n
\end{array}\right)[\varphi(i)]^{n}\left[(1-\varphi(i)]^{N-n}\right. \\
\text { se } n \neq \psi \text { e } a=0 \text { ou } a=1 \\
1 \quad \text { se } n=\psi \text { e } a=0 \\
0 \quad \text { caso contrário }
\end{array}\right.
$$

para todo $i \in E_{I}, n \in E_{\Phi}$.

A estrutura de custos do modelo inclui:

- um custo de operação $C_{O}(i)$, incorrido quando o equipamento produz um lote de peças em um nível de deterioração $i \in E_{l}$;

- um custo de revisão $C_{R}(i)$, incorrido quando, estando o equipamento operando em um nível de deterioração $i \in E_{l}$, decide-se pela revisão do equipamento;

- um custo fixo de produção de peça defeituosa $C_{L}$, incorrido cada vez que uma peça defeituosa é produzida.

Para simplicidade do modelo e sem perda de generalidade, o custo de inspeção de um lote será considerado como parte do custo de operação. Assim como quando ocorre uma revisão, considera-se, como parte do custo de revisão, um custo de perda de oportunidade (por não estar produzindo).

O valor esperado do custo total incorrido até o próximo instante de observação é obtido pela soma das parcelas de custos correspondentes aos custos de operação, de revisão e de peças defeituosas. Quando se decide manter o equipamento funcionando $(a=0)$ dada uma observação $n \in E_{\Phi}$, incorre-se em um custo fixo de operação do equipamento $C_{O}(i)$ e em um custo de peças defeituosas cujo valor esperado é o produto da constante de custo de produção de uma peça defeituosa $C_{L}$ pelo número esperado de peças defeituosas produzidas no nível corrente de deterioração, ou seja, pela esperança matemática de uma distribuição binomial com parâmetros $N$ e $\varphi(i)$. Quando se decide revisar o equipamento $(a=1)$, incorre-se somente em um custo fixo de revisão $C_{R}(i)$, dependente do nível de deterioração do equipamento. Desta forma: 


$$
c_{i}(a)=\left\{\begin{array}{lll}
C_{O}(i)+N C_{L} \varphi(i) & \text { se } & a=0 \\
C_{R}(i) & \text { se } & a=1
\end{array}\right.
$$

O objetivo deste modelo é obter uma política de manutenção que decida sobre revisar ou não o equipamento, após a produção de cada lote, de forma a minimizar o custo médio a longo prazo.

\section{Implementação do Modelo}

A implementação do modelo foi feita utilizando-se uma biblioteca de classes para tratamento analítico de modelos markovianos denominada MODESTO - Modelos Estocásticos - em linguagem $\mathrm{C}++$, desenvolvida por ANDRADE et al. (1996). Para o desenvolvimento deste trabalho, o Algoritmo HL utilizado para Processos Markovianos de Decisão com Informação Parcial (apresentado em LOEVE, 1995) foi implementado e incorporado a esta biblioteca de classes. Através deste software, além de se obter resultados do Algoritmo de Iteração de Valores, como a política ótima e o custo médio a longo prazo, obtêm-se algumas medidas de performance do modelo, tais como: fração do tempo em operação, nível médio de deterioração do equipamento, número médio de peças defeituosas por lote etc.

$\mathrm{O}$ algoritmo HL é baseado no algoritmo de iteração de valores e busca uma "boa" política dentro da classe das políticas markovianas admissíveis para a qual o custo médio a longo prazo esperado seja minimizado. $\mathrm{Na}$ sua construção utiliza-se o conceito de quase otimalidade onde uma política periódica admissível é quase ótima quando as derivadas direcionais do custo a ela associadas são positivas para qualquer direção viável. Em LOEVE (1995) pode-se obter mais informações sobre o conceito de quase otimalidade de políticas markovianas admissíveis que será a base para o algoritmo proposto.

Este algoritmo considera dois espaços de estados. O primeiro é o espaço de estados interno do sistema, composto de estados não observáveis, e o segundo é o espaço de observações, a partir do qual as ações serão tomadas. Assim, em um instante de decisão, a única informação disponível é a observação do número de peças defeituosas, $n$, pertencente ao espaço de observações. Isto significa dizer que o estado interno exato do sistema não é conhecido, mas sim uma observação que está relacionada com o estado interno não observável.

$\mathrm{O}$ algoritmo HL, para um Processo Markoviano de Decisão com Informação Parcial, é descrito a seguir. Neste algoritmo, para obter o custo esperado mínimo total, calcula-se recursivamente para $k=1,2, \ldots$, o valor da função de custo relativo $V_{i}^{k}$, onde:

$$
\begin{aligned}
& V_{i}^{k}=\min _{a \in A(i)} q_{i n} x_{i}^{k}\left\{c_{i}(a)+\sum_{j \neq i} p_{i j}(a) V_{j}^{k-1}\right\}, \\
& i, j \in E_{I} \text { e } n \in E_{\Phi}
\end{aligned}
$$

começando com uma função arbitrariamente escolhida $V_{i}^{0}, i \in E_{I}$. Nesta última equação $x^{k}$ é uma estimativa do vetor de probabilidades limites do processo sob a política de controle corrente e $q_{\text {in }}$, independente do tempo, denota a probabilidade de se observar $n \in E_{\Phi}$ dado que o estado interno é $i \in E_{I}$.

Através da equação $V_{i}^{k}$ pode-se determinar a melhor ação para qualquer estado. Seja a política estacionária definida como uma função do processo de observação. Assim, $f(n)$ é a ação escolhida quando a observação é $n \in E_{\Phi}$. Para $i, j \in E_{I}$, as estruturas da matriz de transição e do custo do sistema são dadas por:

$$
\begin{aligned}
& P_{i j}(f)=\sum_{n=1}^{N} q_{i n} p_{i j}(f(n)) \\
& c_{i}(f)=\sum_{n=1}^{N} q_{i n} c_{i}(f(n))
\end{aligned}
$$

Os valores do vetor de probabilidades, $x^{k}$, e do vetor de custo, $V^{k}$, serão atualizados considerando as equações acima.

$\mathrm{O}$ algoritmo HL para um Processo Markoviano de Decisão com Informação Parcial, para o 
caso discreto foi implementado seguindo os seguintes passos:

1) Escolher uma regra de decisão determinística admissível, $f^{0}$, e a precisão desejada, $\varepsilon>0$.

2) Escolher o vetor de probabilidade, $x^{l}$, e o vetor de custo, $V^{l} \in \mathfrak{R}^{N}$.

3) Fazer para $k$ iterações $(k=1,2,3, \ldots .)\{$.

$\mathbf{g}^{k}(n, a)=\sum_{i=1}^{D} q_{i n} x_{i}^{k}\left\{c_{i}(a)+\sum_{j=1}^{D} p_{i j}(a) V_{j}^{k}\right\}, a \in A$.

Para $n=1,2, \ldots, N$

se $f^{k-1}(n) \in \arg \min _{a \in A} \mathrm{~g}^{k}(n, a)$ então $f^{k}(n)=f^{k-1}(n)$, ou seja, se a política encontrada for igual à inicial manter a política inicial;

caso contrário, a nova política será $f^{k}(n)=a \quad$ para algum $a \in \arg \min _{a \in A} \mathbf{g}^{k}(n, a)$;

$$
\begin{aligned}
x^{k+1} & =x^{k} P\left(f^{k}\right) ; \\
V^{k+1} & =c\left(f^{k}\right)+P\left(f^{k}\right) V^{k}
\end{aligned}
$$

\}

O algoritmo pára se para algum $L$ e $m=k+1-L$ é assegurado que:

- $f^{m+L-1}=f^{m-1}$,

- $\left\|x^{m+L}-x^{m}\right\|<\varepsilon$, onde $\|x\|<\varepsilon$ para $x \in \mathfrak{R}^{N}$ significa que $\left|x_{i}\right|<\varepsilon \forall i$ e

- $\operatorname{span}\left(V^{m+L}-V^{m}\right)<\varepsilon$, onde span $=\max \left(V^{m+L}-V^{m}\right)-\min \left(V^{m+L}-V^{m}\right)$

Se o algoritmo pára para algum $m$ e $L$, é escolhida a política periódica $\left(f^{m+L-1}, f^{m+L-2}, \ldots, f^{m}\right)^{\infty}$.

\section{Resultados Numéricos}

$\mathrm{O}$ dados considerados como exemplos refletem o comportamento de um equipamento que desde o início de sua atividade sofre um processo de deterioração crescente. As probabilidades e custos (descritos na Tabela 1), que estão diretamente relacionadas aos níveis de deterioração do equipamento, também são crescentes.
Na implementação computacional, consideraram-se os seguintes dados fixos:

- Nível máximo de deterioração: $D=10$

- Tamanho do lote: $N=20$

- Custo por peça defeituosa: $C_{L}=1,5$

Os dados que variam de acordo com o nível de deterioração do equipamento são apresentados na Tabela 1.

Por hipótese, será considerado que o equipamento só pode deteriorar um nível de cada vez. Assim, por exemplo, pela Tabela 1, no nível de deterioração 0 , o equipamento tem $90 \%$ de chance de não se deteriorar, isto é, de permanecer no mesmo nível de deterioração e $10 \%$ de se deteriorar, isto é, de ir para o nível de deterioração 1.

Com respeito à probabilidade de produção de peças defeituosas, dado que o modelo descrito não admite quebra do equipamento, foi suposto que no último nível de deterioração todas as peças produzidas pelo equipamento são defeituosas com probabilidade 1 .

No modelo consideram-se os custos de revisão, de operação e a probabilidade de produção de peça defeituosa crescentes em relação ao nível de deterioração. Os custos de revisão foram considerados crescentes porque foi suposto que, mesmo não se considerando quebra do equipamento, as peças trocadas nas revisões com níveis de deterioração baixos são mais baratas ou em menor quantidade do que as peças trocadas em revisões com níveis de deterioração mais altos. Com respeito ao custo de operação, os dados foram considerados crescentes por se considerar mais caro operar em níveis de deterioração elevados.

$\mathrm{Na}$ Tabela 2 apresentam-se os resultados obtidos, de acordo com os dados fornecidos. Apresentam-se, ainda, resultados do modelo denominado modelo com informação total. Este modelo considera que o nível de deterioração do equipamento é conhecido em todos os instantes de decisão, e é utilizado para avaliar a política de manutenção obtida pelo modelo com informação parcial. Isso de deve ao fato que os custos obtidos no modelo com informação total são um limitante inferior para os custos obtidos no 
Tabela 1 - Dados de entrada do modelo.

\begin{tabular}{|c|c|c||c|c|}
\hline $\begin{array}{c}\text { Nível de } \\
\text { deterioração }(d)\end{array}$ & $\begin{array}{c}\text { Prob. de } \\
\text { deterioração }\left(p_{i j}\right)\end{array}$ & $\begin{array}{c}\text { Prob. de prod. } \\
\text { peça def. }(\varphi)\end{array}$ & $\begin{array}{c}\text { Custo de revisão } \\
\left(C_{R}\right)\end{array}$ & $\begin{array}{c}\text { Custo de } \\
\text { operação }\left(C_{O}\right)\end{array}$ \\
\hline 0 & 0,10 & 0,10 & 230 & 5 \\
1 & 0,15 & 0,15 & 240 & 10 \\
2 & 0,20 & 0,20 & 250 & 15 \\
3 & 0,25 & 0,25 & 260 & 20 \\
4 & 0,30 & 0,30 & 270 & 25 \\
5 & 0,35 & 0,40 & 280 & 30 \\
6 & 0,40 & 0,50 & 290 & 35 \\
7 & 0,45 & 0,60 & 300 & 40 \\
8 & 0,50 & 0,70 & 310 & 45 \\
9 & 0,55 & 0,80 & 320 & 50 \\
10 & 0,00 & 1,00 & 350 & 75 \\
\hline
\end{tabular}

Tabela 2 - Resultados dos modelos com informação parcial e informação total - custo de operação crescente.

\begin{tabular}{|c|c|c|}
\hline & Modelo com Inf. PARCIAL & Modelo com Inf. TOTAL \\
\hline Custo Total & 25,9926 & 24,8529 \\
\hline Custo de Operação & 9,8512 & 9,4117 \\
\hline Custo de Revisão & 12,0387 & 11,4706 \\
\hline Custo de Prod. Pç. Def. & 4,1027 & 3,9706 \\
\hline Período Ótimo & 4 & 1 \\
\hline \multirow[t]{3}{*}{ Política Ótima } & $4 x\{0\}$ & $d \leq 2$ \\
\hline & $3 x\{1\} 1 x\{0\}$ & $d \geq 3$ \\
\hline & $n \geq 8 \quad 4 x\{1\}$ & \\
\hline Nível Médio de Det./ tempo & 1,0094 & 0,7692 \\
\hline Fração Tempo em Operação & 0,9536 & 0,9559 \\
\hline № ${ }^{\mathrm{o}}$ Médio de Pç. Def./ lote & 3,0179 & 2,7692 \\
\hline
\end{tabular}

modelo com informação parcial. O algoritmo utilizado no modelo com informação total para obter o custo esperado mínimo total é o Algoritmo de Iteração de Valores descrito em TIJMS (1994).
Pela Tabela 2 observa-se que o custo total no modelo com informação total é menor do que o obtido no modelo com informação parcial. Isto era esperado, pois no modelo com informação total as decisões sobre revisar ou não o 


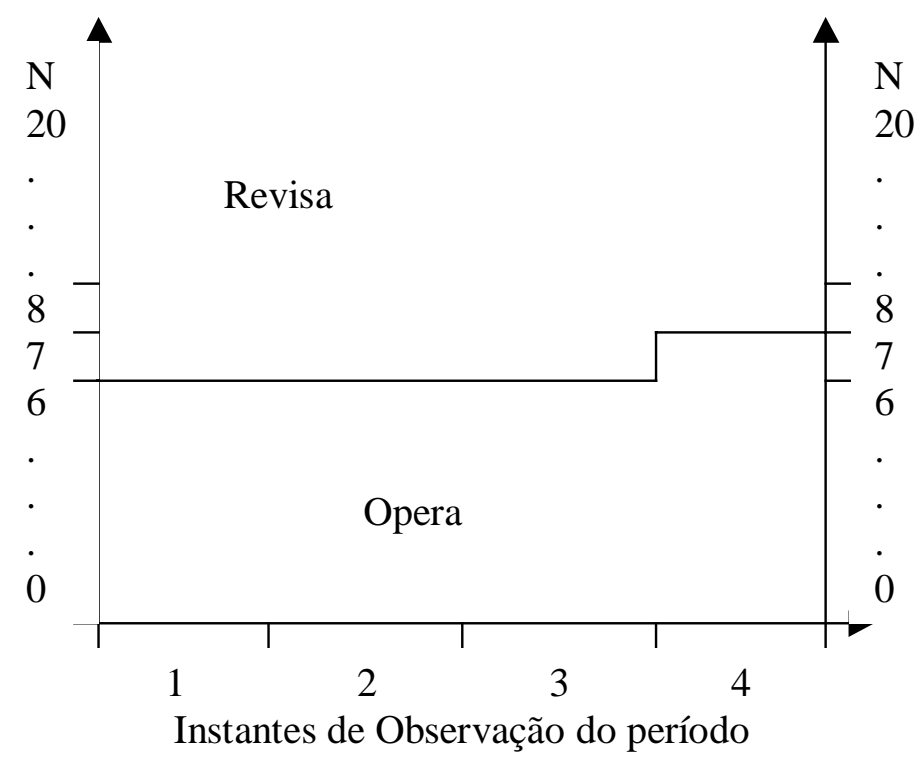

Figura 1 - Política obtida para o modelo com informação parcial.

equipamento são baseadas no nível de deterioração do equipamento $(d)$, enquanto que no modelo com informação parcial as decisões são baseadas no número de peças defeituosas $(n)$ fornecido pela inspeção. Assim, no modelo com informação parcial obteve-se um custo esperado médio a longo prazo $4,6 \%$ maior que o custo obtido no modelo com informação total.

O período ótimo, apresentado na Tabela 2, está relacionado à política do modelo com informação parcial que é do tipo periódica. Neste caso, o algoritmo encontrou uma política periódica admissível igual a 4. Isto significa que para um dado instante de observação, se o número de peças defeituosas observado for menor ou igual a 6 , qualquer que seja o período da política (1, 2, 3 ou 4), a ação escolhida será 0 (continuar operando). Se o número de peças defeituosas observado for qualquer valor maior ou igual a 8 , qualquer que seja o período da política $(1,2,3$ ou 4), a ação escolhida será 1 (revisar). Mas, se o número de peças defeituosas observado for 7 , o período da política influenciará na ação a ser escolhida. Neste caso, para $n=7$, se o período for 1,2 ou 3 a ação será $O$ (continuar operando) e se o período for 4 a ação será revisar o equipamento. Assim, no modelo com informação parcial, a decisão sobre revisar ou continuar a produzir dependerá do número de peças defeituosas encontrado e do instante de observação do período, como mostra a Figura 1.

No caso do modelo com informação total a política é baseada no nível de deterioração do equipamento $(d)$. Assim, se o nível de deterioração for menor ou igual a 2, a ação a ser tomada será 0 (continuar operando). Caso contrário, a ação será 1 (revisar o equipamento). Ressalta-se, neste caso, que a política não leva em consideração a informação fornecida pelo modelo sobre o número de peças defeituosas produzido. A política neste tipo de modelo possui sempre periódica igual a 1 .

A fração de tempo em operação, fornecida pelos modelos, mostra que eles passam praticamente o mesmo tempo em operação. Para o modelo com informação total, $95,6 \%$ do tempo de sua vida útil é gasto operando entre os níveis 0 a 2 de deterioração e produzindo em média 2,77 peças defeituosas por lote. No modelo com informação parcial tem-se que, da fração do tempo em que o equipamento passa operando $(95,4 \%)$, este produz em média 3,02 peças defeituosas por lote. 
Tabela 3 - Resultados dos modelos com informação parcial e informação total - custo de operação constante.

\begin{tabular}{|c|c|c|}
\hline & Modelo com Inf. PARCIAL & Modelo com Inf. TOTAL \\
\hline Custo Total & 49,3411 & 49,1333 \\
\hline Custo de Operação & 34,8159 & 34,8000 \\
\hline Custo de Revisão & 9,2847 & 9,3333 \\
\hline Custo de Prod. Pç. Def. & 5,2405 & 5,0000 \\
\hline \multirow[t]{2}{*}{ Política Ótima } & $n \leq 9$ & $d \leq 4$ \\
\hline & $n \geq 10$ & $d \geq 5$ \\
\hline Nível Médio de Det./ tempo & 1,7333 & 1,4483 \\
\hline Fração Tempo em Operação & 0,9671 & 0,9667 \\
\hline №̣ Médio de Pç. Def./ lote & 3,8349 & 3,4483 \\
\hline
\end{tabular}

\section{Outros Casos Considerados}

\section{a) Custo de operação constante}

A seguir apresentam-se os resultados obtidos considerando o custo de operação constante (e igual a 35) para todos os níveis de deterioração. Note-se que este valor é a média obtida com os valores do custo de operação apresentados na Tabela 1. Os demais dados de entrada permanecem inalterados.

Assim como na Tabela 2, o resultado obtido considerando o custo de operação constante (apresentado na Tabela 3) levou a um custo médio a longo prazo maior para o modelo com informação parcial, em $0,42 \%$, em relação ao modelo com informação total.

Comparando os resultados das Tabelas 2 e 3 , para os dois tipos de custo de operação, verificase que, para os dados de entrada considerados (lembrar que a média dos valores dos custos de operação é a mesma), o menor custo médio a longo prazo foi obtido quando o custo de operação foi considerado crescente. Isto, de certa forma, era esperado, pois neste caso o equipamento permanece mais tempo em níveis de deterioração baixos e, portanto, com custo de operação menor. Verifica-se também que, quanto maior o valor do ponto de início das revisões, maior é a fração do tempo em operação do equipamento e maior é o número médio de peças defeituosas produzidas por lote, como seria de se esperar.

Uma das medidas de desempenho fornecida pelo software para os modelos com informação parcial e total é a fração do tempo em operação em cada nível de deterioração. As Figuras 2 e 3 ilustram o comportamento desta medida de desempenho nos modelos com informação parcial e total para os dois tipos de custo de operação considerados.

Verifica-se que a fração do tempo em operação em cada nível de deterioração decresce quando o nível de deterioração aumenta; isto significa que a probabilidade do equipamento operar em níveis elevados de deterioração é menor do que nos níveis baixos de deterioração. Portanto, quanto maior o nível de deterioração em que o equipamento se encontra, maior a probabilidade de ocorrer revisões.

Outra medida de desempenho fornecida pelo software é a probabilidade de produzir peças defeituosas por lote. A Figura 4 apresenta, para o modelo com informação parcial, a probabilidade de se produzir peças defeituosas por lote para cada um dos dois tipos de custo de operação. 


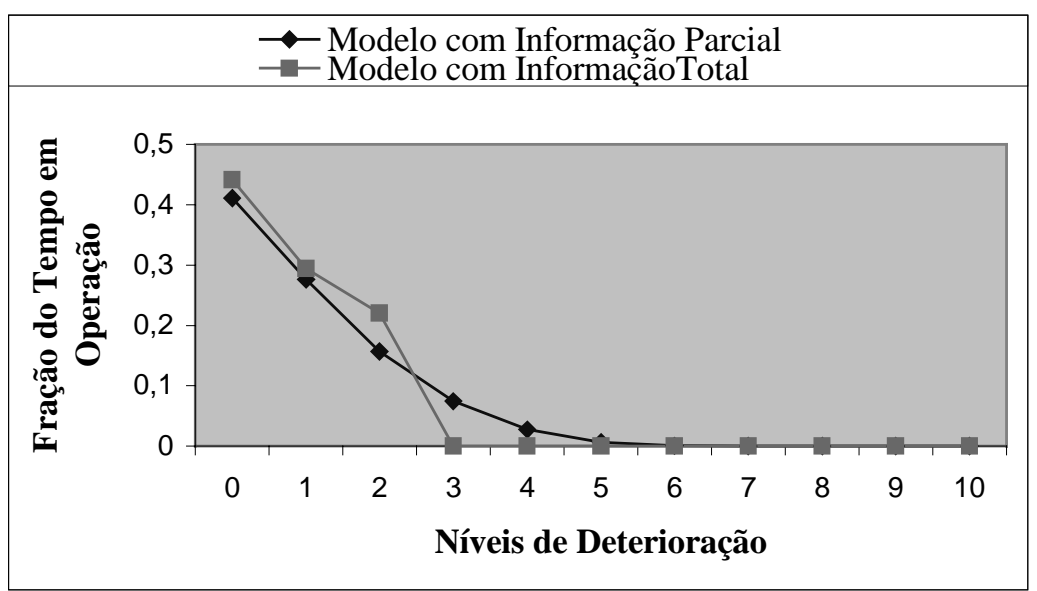

Figura 2 - Fração do tempo em operação em cada nível de deterioração - modelo com custo de operação crescente.

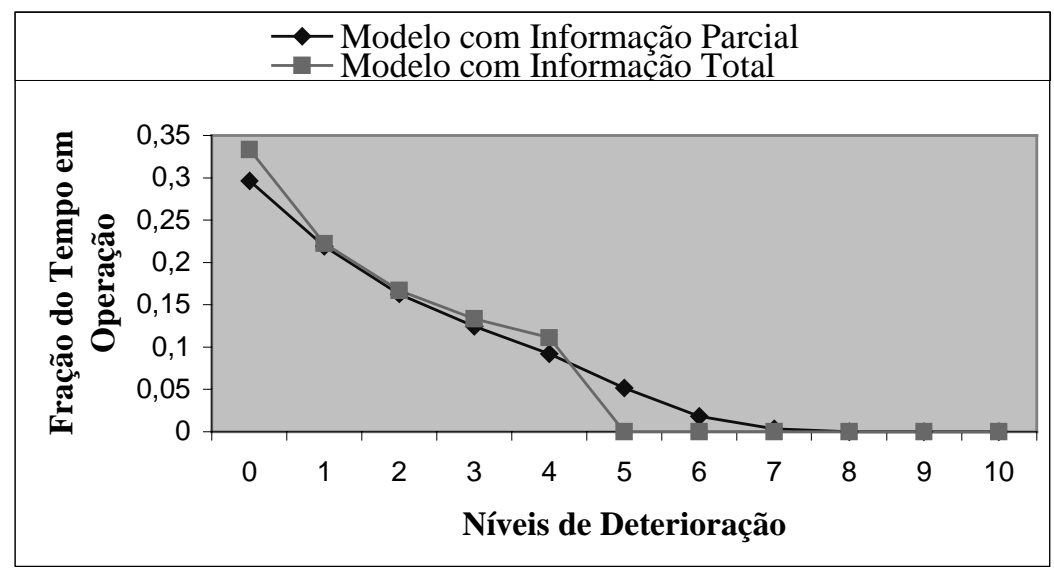

Figura 3 - Fração do tempo em operação em cada nível de deterioração - modelo com custo de operação constante.

Para estes valores, tem-se que a probabilidade de ocorrer revisões é de $10,5 \%$ para o modelo com custo de operação crescente e de $3,4 \%$ para o modelo com custo de operação constante. Com isto, pode-se concluir que o modelo no qual ocorrerão mais revisões ao longo do tempo, em média, será o modelo com custo de operação crescente.

\section{b) Variando o custo de revisão}

Para analisar o comportamento dos modelos com informação parcial e total, variou-se também o custo de revisão. Foram consideradas variações de $-50 \%$ a $150 \%$ em relação aos custos de revisão apresentados na Tabela 1 , sendo que os demais dados permaneceram inalterados. Os custos e políticas obtidos para cada modelo encontram-se discriminados na Tabela 4 a seguir.

Pela Tabela 4, verifica-se que, quando aumenta o custo de revisão, o nível de deterioração (no caso do modelo com informação total) e o número de peças defeituosas observado (no caso do modelo com informação parcial) em que as revisões ocorrem também aumentam, ou seja, as 


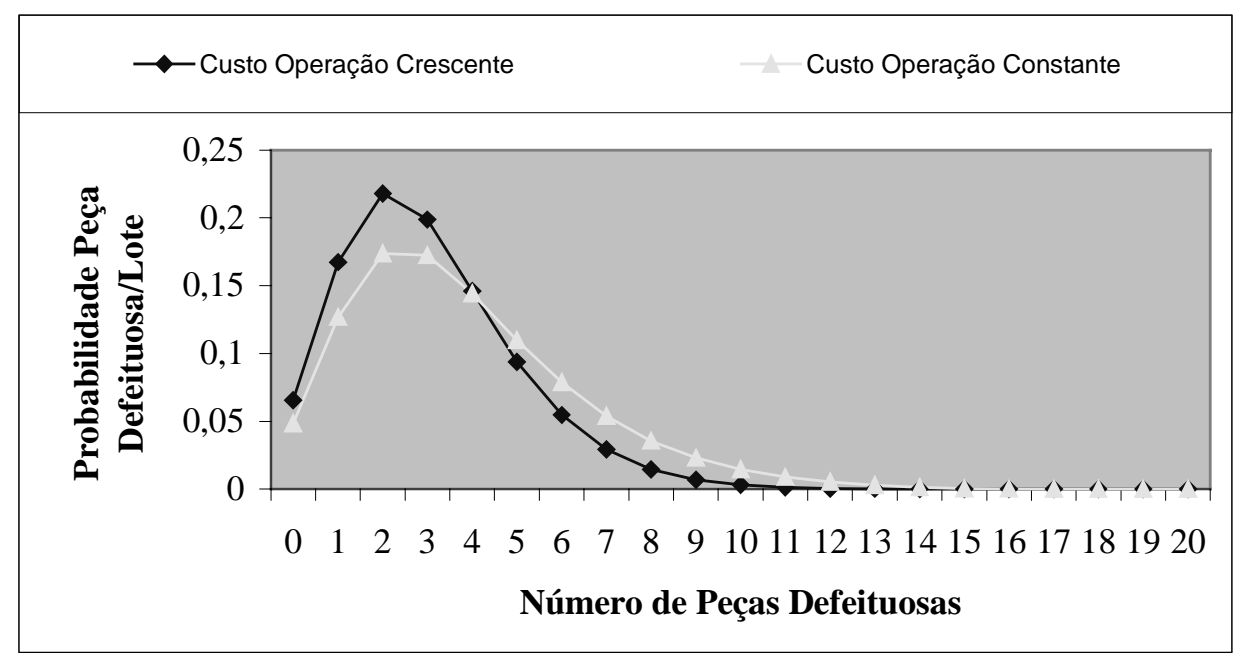

Figura 4 - Probabilidade de produzir peças defeituosas/lote no modelo com informação parcial.

Tabela 4 - Custo total e política para diferentes custos de revisão.

\begin{tabular}{|c|c|c|c|c|c|c|}
\hline \multirow{2}{*}{$\begin{array}{l}\text { Variação nos } \\
\text { Custos Revisão }\end{array}$} & \multicolumn{4}{|c|}{ Política Ótima } & \multicolumn{2}{|c|}{ Custo Total } \\
\hline & \multicolumn{2}{|c|}{ Inf. Total } & \multicolumn{2}{|c|}{ Inf. Parcial } & Inf. Total & Inf. Parcial \\
\hline$-50 \%$ & $\begin{array}{l}d \leq 1 \\
d \geq 2\end{array}$ & $\begin{array}{l}\{0\} \\
\{1\}\end{array}$ & $\begin{array}{l}n \leq 5 \\
n=6 \\
n \geq 7\end{array}$ & $\begin{array}{r}4 \times\{0\} \\
3 \times\{1\} 1 \times\{0\} \\
4 x\{1\}\end{array}$ & 18,0189 & 19,5026 \\
\hline $0 \%$ & $\begin{array}{l}d \leq 2 \\
d \geq 3\end{array}$ & $\begin{array}{l}\{0\} \\
\{1\}\end{array}$ & $\begin{array}{l}n \leq 6 \\
n=7 \\
n \geq 8\end{array}$ & $\begin{array}{r}4 \times\{0\} \\
3 \times\{1\} 1 \times\{0\} \\
4 \times\{1\}\end{array}$ & 24,8529 & 25,9926 \\
\hline $50 \%$ & $\begin{array}{l}d \leq 2 \\
d \geq 3\end{array}$ & $\begin{array}{l}\{0\} \\
\{1\}\end{array}$ & $\begin{array}{l}n \leq 7 \\
n \geq 8\end{array}$ & $\begin{array}{l}\{0\} \\
\{1\}\end{array}$ & 30,5882 & 31,5793 \\
\hline $100 \%$ & $\begin{array}{l}d \leq 3 \\
d \geq 4\end{array}$ & $\begin{array}{l}\{0\} \\
\{1\}\end{array}$ & $\begin{array}{l}n \leq 7 \\
n=8 \\
n \geq 9\end{array}$ & $\begin{array}{r}8 x\{0\} \\
7 \times\{0\} 1 \times\{1\} \\
8 x\{1\}\end{array}$ & 35,9000 & 36,7760 \\
\hline $150 \%$ & $\begin{array}{l}d \leq 3 \\
d \geq 4\end{array}$ & $\begin{array}{l}\{0\} \\
\{1\}\end{array}$ & $\begin{array}{l}n \leq 8 \\
n=9 \\
n \geq 10\end{array}$ & $\begin{array}{r}4 \times\{0\} \\
3 \times\{1\} 1 \times\{0\} \\
4 \times\{1\}\end{array}$ & 40,9625 & 41,6820 \\
\hline
\end{tabular}

revisões passam a ocorrer mais tarde, como esperado. No caso do modelo com informação total, revisões que ocorrem a partir do nível de deterioração 2 quando o custo de revisão é $50 \%$ menor, passam a ocorrer a partir do nível de deterioração 4 quando o custo de revisão é $150 \%$ maior. No caso do modelo com informação parcial, o número máximo de peças defeituosas observadas para o equipamento continuar operando também aumenta, considerando estes dois casos, de 5 para 8 .

Verifica-se também na Tabela 4 que os custos totais nos dois modelos se aproximam quando os valores do custo de revisão aumentam e os demais dados de entrada do modelo permanecem inalterados. Como já ressaltado anteriormente, quando o número de peças defeituosas para o início das revisões aumenta, maior é a fração do 
tempo em operação do equipamento. Isto parece indicar que, quanto maior o tempo de funcionamento do equipamento, menor é a diferença entre os modelos com informação parcial e total.

\section{Comentários Finais}

$\mathrm{E}$ ste trabalho teve como principal enfoque apresentar uma nova abordagem na análise de modelos de manutenção de equipamentos quando não se pode observar diretamente os níveis de deterioração do equipamento. Esta nova abordagem utiliza o número de peças defeituosas encontradas em um lote produzido de peças para se tomar decisões acerca da manutenção do equipamento. Ainda, utilizou-se uma técnica recente de modelagem de sistemas, a modelagem por Processos Markovianos de Decisão com Informação Parcial. Testes numéricos foram realizados e comparados com um modelo com informação total (neste tipo de modelo considera-se que os níveis de deterioração do equipamento podem ser observados).

No caso do modelo com informação parcial, a única informação disponível nos momentos de tomada de decisão é o número de peças defeituosas encontradas em cada lote inspecio- nado. Neste caso, admite-se que existe uma probabilidade associando o número de peças defeituosas encontrada em um lote com o estado de deterioração do equipamento.

No modelo com informação total, por outro lado, trabalha-se com a hipótese de que no momento de se decidir que ação tomar, conhecese com certeza o nível de deterioração do equipamento.

Apesar de não ter sido considerado neste trabalho um exemplo de modelo onde não se tem informações sobre o nível de deterioração do equipamento, é possível obter políticas que minimizem o custo médio a longo prazo por meio do modelo com informação parcial considerando esta hipótese. Isto é possível pois este trabalha com informações relacionadas à observação da produção do equipamento e não com o seu estado de deterioração, como é o caso do modelo com informação total.

Uma possível extensão deste trabalho seria considerar que a inspeção é imperfeita. Neste caso, a informação fornecida pela inspeção de um lote pode estar incorreta, isto é, uma peça boa pode ser considerada defeituosa e uma peça defeituosa pode ser considerada boa. Este caso será considerado num trabalho futuro.

\section{Referências Bibliográficas}

ANDRADE V.M.B.; CARVALHO, S.V. \& VIJAYKUMAR, N.L.: "Desenvolvimento de um software para análise de sistemas através de modelos markovianos - uma abordagem orientada a objetos". São José dos Campos, Instituto Nacional de Pesquisas Espaciais (Relatório interno do INPE/LAC), 1996.

CASSANDRA A.R.: "Optimal policies for partially observable Markov decision processes". CS-94-14, Department of Computer Science, Brown University, 1994.

HORDIJK, A. \& LOEVE, J.A.: "Undiscounted Markov decision chains with partial information: an algorithm for computing a locally periodic policy". ZOR - Mathematical Methods of Operational Research, v.40, p.163-181, 1994.
GONG, L. \& TANG, K.: "Monitoring machine operations using on-line sensors". European Journal of Operational Research, v.96, p.479-492, 1997.

LOEVE, J.A.: Markov decision chains with partial information. Tese (Doutorado em Matemática) Universidade de Leiden, Holanda, 1995.

LOVEJOY, W.S.: "A survey of algorithmic methods for partially observed Markov decision processes". Annals of Operations Research, v.28, p.47-66, 1991.

LUSS, H.: "Maintenance policies when deterioration can be observed by inspections". Operations Research, v.24, n.2, p.359-366, 1976.

MILIONI, A.Z. \& PLISKA, S.R.: "Optimal inspection under semi-Markovian deterioration: the catastrophic case". Naval Research Logistics, v.35, p.393-411, 1988. 
MONAHAN G.E.: “A survey of partially observable Markov decision processes theory, models and algorithms". Management Science, v.28, n.1, p.1-16, 1982.

OZEKICI, S. \& PLISKA, S.R.: “Optimal scheduling of inspections: a delayed Markov model with false positives and negatives". Operations Research, v.39, n.2, p.261-273, 1991.

ROSENFIELD, D.: "Markovian deterioration with uncertain information". Operations Research, v.24, n.1, p.141-155, 1976 a.

ROSENFIELD, D.: "Markovian deterioration with uncertain information - a more general model". Naval Research Logistics Quarterly, v.23, n.3, p.389-405, 1976 b.
ROSS, S.M.: "Quality control under Markovian deterioration". Management Science, v.17, n.9, p.587-596, 1971.

SMALLWOOD, R.D. \& SONDIK, E.J.: "The optimal control of partially observable Markov processes over a finite horizon". Operations Research, v.21, n.5, p.1071-1088, 1973.

TAPIERO, C.S.: "Continuous quality production and machine maintenance”. Naval Research Logistics Quarterly, v.33, n.3, p.489-499, 1987.

TIJMS, H.C.: Stochastic modelling and analysis: a computational approach. John Wiley \& Sons, New York, 1994.

\title{
A STOCASTIC MODEL FOR A MAINTENANCE SYSTEM BASED ON THE INSPECTION OF THE PRODUCED PARTS
}

\begin{abstract}
In this paper a maintenance model is presented for an equipment deteriorating with time. The equipment periodically produces a batch of parts. Each batch is inspected and based on the number of defective parts, a decision about the revision of the equipment is made. The goal of the model is to obtain a maintenance policy for the equipment that minimizes the long run average cost. The cost structure includes an operation cost, a revision cost and a defective part cost. To obtain a minimum cost policy, the problem is modeled as a Markov Decision Process with Partial Information. Numerical examples are presented.
\end{abstract}

Key words: maintenance system, revision policy, Markov decision process with partial information. 